\title{
焙烧处理下二氧化钛/钛酸盐纳米材料晶型和形貌的变化规律研究
}

\author{
赵斌*, 林琳 ${ }^{a} \quad$ 陈超 ${ }^{a} \quad$ 柴瑜超 $^{b} \quad$ 何丹农 ${ }^{a, b}$ \\ ( ${ }^{a}$ 纳米技术及应用国家工程研究中心 上海 200241) \\ ( ${ }^{b}$ 上海交通大学材料科学与工程学院 上海 200240)
}

\begin{abstract}
摘要 通过调控酸碱浓度, 在水热条件下得到了金红石、锐钛矿、板钛矿、钛酸钠等一系列 $\mathrm{TiO}_{2} /$ 钛酸盐产物. 对上述 $\mathrm{TiO}_{2}$ 、酸洗处理后的钛酸盐等一系列不同晶型、不同形貌的样品进行焙烧处理, 系统性地研究焙烧温度的逐渐升高对 产物晶型转变和形貌演化的规律性影响. 给出了水热酸碱浓度以及焙烧温度两个因素与 $\mathrm{TiO}_{2} /$ 钛酸盐纳米材料晶型和 形貌变化行为关系的二维示意图. 依据奥斯特瓦尔德阶梯规则、经典热力学理论以及定向附着生长机理, 对 $\mathrm{TiO}_{2} /$ 钛酸 产物的晶型晶体生长、晶型转变和形貌演化机理进行了探讨.
\end{abstract}

关键词 二氧化钛; 钛酸盐; 焙烧处理; 晶型转变; 形貌演化; 机理

\section{Research on the Phase Transition and Morphological Evolution Behaviors of Titania/Titanate Nanomaterials by Calcination Treatment}

\author{
Zhao, Bin*,a Lin, $\mathrm{Lin}^{a} \quad \mathrm{Chen}^{*} \mathrm{Chao}^{a} \quad \mathrm{Chai} \mathrm{Yuchao}^{b} \quad \mathrm{He}, \mathrm{Dannong}^{a, b}$ \\ ( ${ }^{a}$ National Engineering Research Center for Nanotechnology, Shanghai 200241) \\ ( ${ }^{b}$ School of Material Science and Engineering, Shanghai JiaoTong University, Shanghai 200240)
}

\begin{abstract}
Titania/titanate nanomaterials including rutile, anatase, brookite $\mathrm{TiO}_{2}$ and sodium dititanate and trititanate were obtained by regulating the acid/alkali concentration under hydrothermal treatment. A systematical investigation was established to uncover the phase transition and morphological evolution behaviors of $\mathrm{TiO}_{2} /$ titanate nanomaterials by calcining the samples including rutile $\mathrm{TiO}_{2}$ nanorods, anatase $\mathrm{TiO}_{2}$ nanocrystallines, brookite $\mathrm{TiO}_{2}$ nanoflowers, acid washed dititanate $\mathrm{H}_{2} \mathrm{Ti}_{2} \mathrm{O}_{5}$ nanosheets and trititanate $\mathrm{H}_{2} \mathrm{Ti}_{3} \mathrm{O}_{7}$ nanowires at $400,600,800$ or $1000{ }^{\circ} \mathrm{C}$ for $4 \mathrm{~h}$ in air with the heating rate of 2 ${ }^{\circ} \mathrm{C} / \mathrm{min}$. After heat-treatment, the products were taken out from the oven and cooled down to the room temperature. Rietveld refinements of the powder X-ray diffraction (XRD) pattern were used to generally assess the phase composition of the different samples and their crystallite sizes, and to further investigate the phase transition behavior in company with the synthetic parameters. FESEM, TEM, and HRTEM were used to characterize the morphology evolution and to further elucidate the morphological evolution of the resulting products. The crystalline phase distributed diagram of $\mathrm{TiO}_{2} /$ titanate nanostructures dominated by the two experimental parameters indcluding acid/alkali concentration and calcination temperature was presented in the current work based on our experimental results, in which revealed the 5 types of phase transition and morphological evolution behaviors of titania/titanate nanomaterials. 1. Rutile nanorods $\rightarrow$ rutile nanorod aggregations $\rightarrow$ rutile micro particles. 2. Anatase nanocrystallines $\rightarrow$ anatase nanoparticle aggregations $\rightarrow$ rutile micro particles. 3. Brookite nanoflowers $\rightarrow$ brookite nanoflower clusters $\rightarrow$ rutile micro clusters. 4. Dititanate $\mathrm{H}_{2} \mathrm{Ti}_{2} \mathrm{O}_{5}$ nanosheets $\rightarrow$ anatase nanoparticle aggregations $\rightarrow$ rutile micro clusters. 5. Trititanate $\mathrm{H}_{2} \mathrm{Ti}_{3} \mathrm{O}_{7}$ nanowires $\rightarrow \mathrm{TiO}_{2}-\mathrm{B}$ nanowires $\rightarrow$ anatase nanowire aggregations $\rightarrow$ rutile micro clusters. The crystal growth and phase transition mechanism was discussed based on the Ostwald's step rule. Moreover, morphological evolution mechanism was also discussed based on the thermodynamic equilibrium regime and oriented attachment growth model.
\end{abstract}

Keywords titania; titanate; calcinations; phase transition; morphological evolution; mechanism

\section{1 引言}

在纳米材料研究领域中, $\mathrm{TiO}_{2} /$ 钛酸盐纳米材料的制 备与性能研究具有极为重要的意义. 纳米 $\mathrm{TiO}_{2}$ 具有光
催化能力强、化学稳定性好、抗光腐蚀、安全无毒、无 二次污染、成本不高、原料易得等优点，可用于空气净 化、污水处理、光解水制氢、抗菌、防雾自清洁、太阳 能电池等诸多领域 ${ }^{[1 \sim 6]}$. 而钛酸盐纳米材料由于其独特

*E-mail: zhaobinwily@hotmail.com; Tel.: 0086-021-34291286-8082; Fax: 0086-021-34291125

Received September 27, 2012; published December 10, 2012.

Supporting information for this article is available free of charge via the Internet at http://sioc-journal.cn.

Project supported by the Shanghai International Science and Technology Cooperation Project (No. 11520706100), Shanghai Rising-Star Program (B-type) (No. 12QB1402800), National Natural Science Foundation of China (No. 21071098) and International Science and Technology Cooperation Project of China (No. 2011DFA50530).

项目受上海市国际科技合作基金(No. 11520706100)、上海市青年科技启明星计划(B 类)(No. 12QB1402800)、国家自然科学基金(No. 21071098)以及国 家国际科技合作项目(No. 2011DFA50530)资助. 
的层状结构, 因而具有离子可交换性, 可用于锂离子电 池、催化氧化、离子吸附剂、抗菌涂料、安培电流计、 隔热材料等方面 ${ }^{[7 \sim 10]}$. 而且, 在特定的条件下, $\mathrm{TiO}_{2}$ 和 钛酸盐可以实现晶型的相互转变 ${ }^{[1]}$. 通过人工手段有 效地控制纳米材料的晶型结构、尺寸和形貌对于纳米科 技的发展来说具有深远的意义. 特别是对于 $\mathrm{TiO}_{2} /$ 钣酸 (盐)纳米材料来说, 其电子传导性能、光催化能力等物 化特性强烈地依赖于其晶型、尺寸、形貌和微结构等特 征 ${ }^{[12 ~ 15]}$. 因此, 近年来世界各国都投入了巨大的科技力 量对 $\mathrm{TiO}_{2}$ /钛酸盐纳米材料进行更为系统性的研究工作.

李继光、孙旭东等 ${ }^{[16]}$ 采用不同的氧化剂, 在氧化还 原水热条件下制备了锐钛矿、金红石、板钛矿等一系列 不同晶型的 $\mathrm{TiO}_{2}$ 纳米材料, 并考察了水热体系中反应 物种类以及溶液的 $\mathrm{pH}$ 等条件对产物晶型和形貌的影响. 李继光等 ${ }^{[16]}$ 考察了上述产物的光催化活性, 不同晶型 的 $\mathrm{TiO}_{2}$ 产物在相同比表面积的条件下, 板钛矿纳米片 材料显示出最佳的光催化活性. Morgan 等 ${ }^{[17,18]}$ 报道了不 同 $\mathrm{NaOH}$ 浓度的反应液对铁酸(盐)产物晶型和形貌的影 响. 发现在较低的 $\mathrm{NaOH}$ 浓度下, 产物为层状铁酸盐 $\mathrm{Na}_{2} \mathrm{Ti}_{2} \mathrm{O}_{5}$, 通过酸洗涤可以得到层状钛酸 $\mathrm{H}_{2} \mathrm{Ti}_{2} \mathrm{O}_{5}$ 纳米 管; 在较高的 $\mathrm{NaOH}$ 浓度下, 产物为高级钛酸盐 $\mathrm{Na}_{2} \mathrm{Ti}_{x} \mathrm{O}_{2 x+1}(x \geqslant 3)$ 纳米带. 此外, Morgan 等 ${ }^{[17,18]}$ 还研究 了不同水热温度对产物的影响, 发现较低的水热温度有 利于钛酸(盐)纳米管的生成, 较高的水热温度有利于钛 酸(盐)纳米带的生成. 最终 Morgan 等绘制了水热温度 和碱液浓度对产物晶型与形貌的二维图表. 为 $\mathrm{TiO}_{2} /$ 钛 酸盐纳米材料晶型与形貌的研究提供了一个系统的方 案.

在我们之前的工作中, 我们系统地研究了弱碱水热 体系下 $\mathrm{TiO}_{2}$ /钛酸(盐)纳米材料的晶相转变与形貌演变 规律 ${ }^{[19,20]}$. 此外, 我们设计了一个十分简易的酸碱水热 实验体系. 该实验体系的酸碱浓度范围较宽: 从浓度为 $6 \mathrm{~mol} \cdot \mathrm{L}^{-1}$ 的盐酸到中性溶液, 以及从中性溶液到浓度 为 $10 \mathrm{~mol} \cdot \mathrm{L}^{-1}$ 的 $\mathrm{NaOH}$ 溶液. 通过调控反应体系的酸浓 度或碱浓度, 可以获得金红石、锐钛矿、板钛矿、二钛 钛酸盐和三钛钛酸盐等一系列不同晶型、不同形貌的 $\mathrm{TiO}_{2} /$ 钛酸(盐)纳米材料. 但是, 在上述同一制备体系中 获得的金红石、锐钛矿、板钛矿、二钛钛酸盐和三钛钛 酸盐等一系列 $\mathrm{TiO}_{2}$ /钛酸(盐)纳米材料, 在焙烧处理条件 下的晶型转变以及形貌改变规律尚不清楚, 也未见相关 的研究报道.

因此, 本次工作针对焙烧处理下 $\mathrm{TiO}_{2} /$ 钛酸盐纳米 材料晶型和形貌的变化规律进行研究. 我们对钛酸盐样 品进行了酸洗处理, 得到了相对应的二钛钛酸和三钛钛 酸样品. 之后对金红石、锐钛矿、板钛矿、二钛钛酸和 三钛钛酸等一系列 $\mathrm{TiO}_{2}$ /铁酸产物进行焙烧处理, 研究 焙烧温度的逐渐升高对产物晶型转变和形貌演化的规 律性影响. 并给出了水热酸碱浓度以及焙烧温度两个因
素对 $\mathrm{TiO}_{2} /$ 钛酸盐纳米材料晶型与形貌影响的二维形貌 晶相示意图.

\section{2 结果与讨论}

在 $\mathrm{TiO}_{2} /$ 钛酸盐纳米材料的焙烧处理实验中, 采用 不同的焙烧方式(如空气环境、氮气环境或真空环境等), 不同的焙烧处理时间(如 $1 \mathrm{~h}, 4 \mathrm{~h}, 8 \mathrm{~h}$ 等)，拟或采用不同 的升温方式(如升温速率 $2{ }^{\circ} \mathrm{C} / \mathrm{min}, 10{ }^{\circ} \mathrm{C} / \mathrm{min}$ 等), 也会 对 $\mathrm{TiO}_{2} /$ 钢酸样品的晶型与形貌变化产生不同的影响. 因此, 在进行焙烧处理实验之前, 需要对焙烧方式、焙 烧时间、升温速率等实验条件进行优选.

本次实验工作中, 进行焙烧处理的 $\mathrm{TiO}_{2} /$ 钛酸盐纳 米材料, 为无机材料. 在焙烧处理过程中, 氧气的存在 对上述材料的晶型与形貌变化影响极小. 结合相关文献 报道 ${ }^{[21 ~ 25]}$, 本次工作选择空气环境进行焙烧处理.

在焙烧时间的实验条件优选中，发现焙烧时间过 短, 则材料的晶型转变尚未达到一个较为稳定的状态. 支持信息中的图 S1 是在 $1.0 \mathrm{~mol} \cdot \mathrm{L}^{-1} \mathrm{NaOH}$ 溶液中水热 产物经酸洗处理后在 $800{ }^{\circ} \mathrm{C}$ 下焙烧 $1 \mathrm{~h}$ (图 S1a)、4h(图 $\mathrm{S} 1 \mathrm{~b}$ )、 $8 \mathrm{~h}$ (图 S1c)样品的 XRD 图谱. 分析谱图可知, 焙 烧处理 $1 \mathrm{~h}$ 的样品(图 S1a), 仍有少量的锐铁矿晶型未转 变为金红石晶型. 而焙烧处理 $4 \mathrm{~h}$ 的样品(图 S1b), 已经 转晶为金红石晶型. 焙烧处理 $8 \mathrm{~h}$ 的样品(图 S1c)与 $4 \mathrm{~h}$ 样品的 XRD 谱图几乎保持一致. 此外, 结合文献报 道 ${ }^{[21,22,26]}, 4 \mathrm{~h}$ 的焙烧处理已经能够排除晶型转变不完 全、样品中残留物质(如结合水)等因素的干扰. 因此, 本 次工作选择 $4 \mathrm{~h}$ 焙烧处理的实验条件.

在升温速率的实验条件优选过程中, 如果升温速率 过快, 则层状钛酸材料的脱水过程将会对产物晶型与形 貌转变的造成更大的干扰. 因此, 升温速率不能过快. 结合文献报道 ${ }^{[23 ~ 26]}$, 本次选择 $2{ }^{\circ} \mathrm{C} / \mathrm{min}$ 的升温速率. 在这样的实验条件下, 能够排除钛酸材料在转晶过程中 脱水对实验结果造成的影响.

在优化后的实验条件下, 进行 $\mathrm{TiO}_{2} /$ 钛酸盐纳米材 料的焙烧处理实验. 在空气环境下、以 $2{ }^{\circ} \mathrm{C} / \mathrm{min}$ 的升温 速率进行 $4 \mathrm{~h}$ 的焙烧处理, 可以获得更为科学可靠的实 验数据.

\section{1 晶型转变分析}

在酸碱水热实验体系中, 通过调控水热体系中的酸 碱浓度, 得到一系列不同晶型的 $\mathrm{TiO}_{2} /$ 铁酸盐样品. 支 持信息中的图 S2 和图 S3 显示: 在 $180{ }^{\circ} \mathrm{C}$ 下, 以钛酸正 四丁酯(TBOT)为钛源, 在浓度为 $0 \sim 6 \mathrm{~mol} \cdot \mathrm{L}^{-1}$ 的盐酸、 中性溶液以及浓度为 $0 \sim 10 \mathrm{~mol} \cdot \mathrm{L}^{-1}$ 的 $\mathrm{NaOH}$ 溶液中水 热处理 $24 \mathrm{~h}$, 可以得到金红石、锐钛矿、板钛矿、钛酸 盐等一系列不同晶型的产物. 并且随着盐酸浓度的降低 或者碱液浓度的增加，产物按照金红石、锐钛矿、板钛 矿、钛酸盐的顺序发生晶型转变.

在图 S2 和图 S3 中单一晶型样品的 XRD 图谱汇总 
于图 1 中. 图 $1 \mathrm{a} \sim \mathrm{c}$ 分别是在 $3.00 \mathrm{~mol} \cdot \mathrm{L}^{-1}$ 盐酸、 0.01 $\mathrm{mol} \cdot \mathrm{L}^{-1}$ 盐酸、 $0.10 \mathrm{~mol} \cdot \mathrm{L}^{-1} \mathrm{NaOH}$ 中所得产物的 XRD 图谱, 分别与粉末衍射标准卡片 JCPDS 21-1276, JCPDS 21-1272, JCPDS 29-1360 (图 S4)相对应, 说明产物为金 红石、锐钛矿和板钛矿晶型. 图 $1 \mathrm{~d}$ 是在 $6.00 \mathrm{~mol} \cdot \mathrm{L}^{-1}$ $\mathrm{NaOH}$ 中所得产物的 XRD 图谱. 以往的研究工 作 ${ }^{[8,11,17,18,28]}$ 认为, 在较低浓度的 $\mathrm{NaOH}$ 中水热得到的产 物, 经过酸洗处理之后, 产物为 $\mathrm{H}_{2} \mathrm{Ti}_{2} \mathrm{O}_{5}$. 并且, 图 $1 \mathrm{~d}$ 与 $\mathrm{H}_{2} \mathrm{Ti}_{2} \mathrm{O}_{5}$ 的 XRD 谱图相类似. 因此, 谱图中 $2 \theta \approx 8.9^{\circ}$ 的 衍射峰应该对应于 $\mathrm{Na}_{2} \mathrm{Ti}_{2} \mathrm{O}_{5}$ 的(200)晶面. 图 $1 \mathrm{e}$ 是在 $8.00 \mathrm{~mol} \cdot \mathrm{L}^{-1} \mathrm{NaOH}$ 中所得产物的 XRD 图谱, $2 \theta \approx 10.4^{\circ}$, $15.7^{\circ}, 29.8^{\circ}$ 的衍射峰对应于 $\mathrm{Na}_{2} \mathrm{Ti}_{3} \mathrm{O}_{7}$ (JCPDS 31-1329, 图 S4)的(001), (101), (300)晶面. 最终, 我们认定, 在 $1.00 \mathrm{~mol} \cdot \mathrm{L}^{-1} \mathrm{NaOH}$ 和 $10.00 \mathrm{~mol} \cdot \mathrm{L}^{-1} \mathrm{NaOH}$ 中所得产物 分别是二钛钛酸钠和三钛钛酸钠.

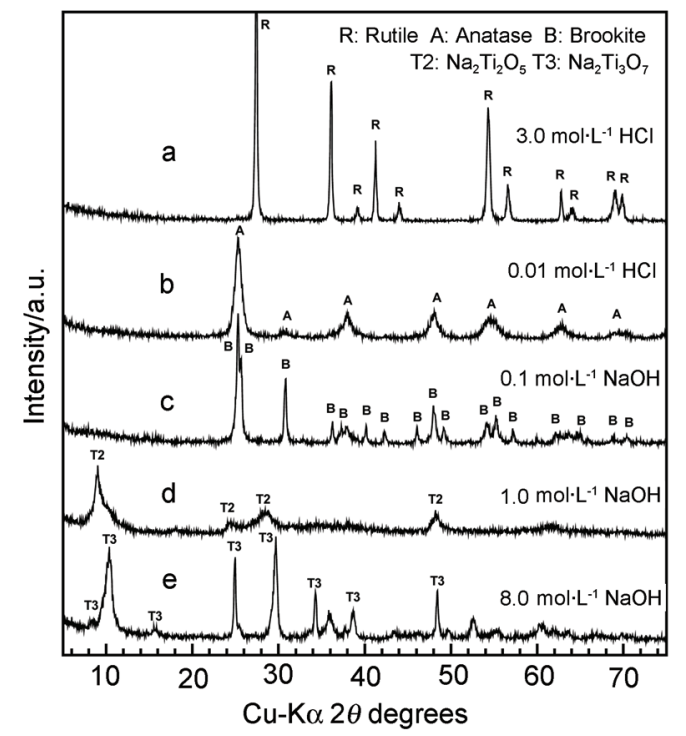

图 1 在(a) $3.0 \mathrm{~mol} \cdot \mathrm{L}^{-1} \mathrm{HCl}$, (b) $0.01 \mathrm{~mol} \cdot \mathrm{L}^{-1} \mathrm{HCl}$, (c) $0.1 \mathrm{~mol} \cdot \mathrm{L}^{-1}$ $\mathrm{NaOH}$, (d) $1.0 \mathrm{~mol} \cdot \mathrm{L}^{-1} \mathrm{NaOH}$ 和(e) $8.0 \mathrm{~mol} \cdot \mathrm{L}^{-1} \mathrm{NaOH}$ 溶液中 $180{ }^{\circ} \mathrm{C}$ 下 水热 $24 \mathrm{~h}$ 所得产物的 XRD 图谱

Figure 1 XRD patterns of products obtained in (a) $3.0 \mathrm{~mol} \cdot \mathrm{L}^{-1} \mathrm{HCl}$, (b) $0.01 \mathrm{~mol} \cdot \mathrm{L}^{-1} \mathrm{HCl}$, (c) $0.1 \mathrm{~mol} \cdot \mathrm{L}^{-1} \mathrm{NaOH}$, (d) $1.0 \mathrm{~mol} \cdot \mathrm{L}^{-1} \mathrm{NaOH}$ and (e) $8.0 \mathrm{~mol} \cdot \mathrm{L}^{-1} \mathrm{NaOH}$ solution at $180{ }^{\circ} \mathrm{C}$ for $24 \mathrm{~h}$ under hydrothermal treatment

我们对上述酸碱水热体系中得到的 5 种典型 $\mathrm{TiO}_{2} /$ 钛酸(盐)样品进行焙烧处理, 考察焙烧温度的逐渐升高 对产物晶型和形貌的规律性影响. 在焙烧处理之前, 我 们对钛酸盐样品进行了酸洗处理, 得到了相对应的二钛 钛酸和三钛钛酸样品. 图 2 4 分别为锐钛矿、板钛矿、 二钛钛酸和三钛钛酸样品在不同的焙烧温度下所得产 物的 XRD 图谱.

众所周知的是, 在所有的 $\mathrm{TiO}_{2}$ 晶型中, 金红石是最 为稳定的晶型, 因此, 在焙烧处理中, 金红石不会产生 晶型的变化 ${ }^{[27]}$. 锐钛矿样品在不同的焙烧温度下晶型 的转变情况见图 $2 \mathrm{a} \sim 2 \mathrm{c}$. 图 $2 \mathrm{a}$ 是在 $0.01 \mathrm{~mol} \cdot \mathrm{L}^{-1}$ 盐酸中 水热所得锐钛矿样品的 XRD 图谱. 并且, 结合支持信
息中的图 S2 和 S3 得知, 锐钛矿样品的 XRD 图谱中, 在 $2 \theta \approx 30.8^{\circ}$ 处的衍射峰与板钛矿的(121)晶面相对应，说 明锐钛矿样品总有极少量的板钛矿晶相的伴生 ${ }^{[16]}$. 在 $600{ }^{\circ} \mathrm{C}$ 下焙烧 $4 \mathrm{~h}$ 后(图 $2 \mathrm{~b}$ ), 样品的 XRD 图谱与图 $2 \mathrm{a}$ 相比, 衍射峰出峰位置无任何变化. 说明 $600{ }^{\circ} \mathrm{C}$ 下焙烧 产物为锐钛矿晶型, 依然有极少量的板钛矿伴生. 但是 锐钛矿主衍射峰的峰强明显增加, 说明在焙烧处理过程 中, 锐钛矿的晶格有序度提高, 即产物的结晶性提高. 在 $800{ }^{\circ} \mathrm{C}$ 下焙烧 $4 \mathrm{~h}$ 后(图 2c), 产物 XRD 图谱与金红石 的粉末衍射标准卡片 JCPDS 21-1276(图 S4)相吻合. 说 明产物在 $800{ }^{\circ} \mathrm{C}$ 的焙烧条件下完全转变为金红石晶型, 无任何其它杂相.

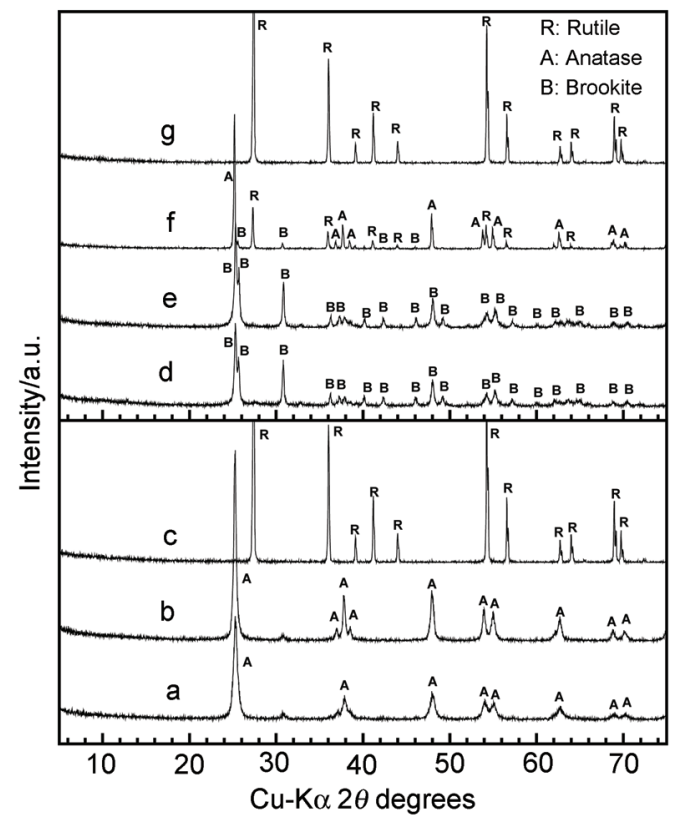

图 2 在 $0.01 \mathrm{~mol} \cdot \mathrm{L}^{-1} \mathrm{HCl}$ 中水热产物(a)未焙烧、(b) $600{ }^{\circ} \mathrm{C}$ 以及(c) $800{ }^{\circ} \mathrm{C}$ 焙烧 $4 \mathrm{~h}$ 样品的 XRD 图谱; 在 $0.1 \mathrm{~mol} \cdot \mathrm{L}^{-1} \mathrm{NaOH}$ 溶液中水热 产物(d)未焙烧、(e) $600{ }^{\circ} \mathrm{C}$ 、(f) $800{ }^{\circ} \mathrm{C}$ 以及 $(\mathrm{g}) 1000{ }^{\circ} \mathrm{C}$ 焙烧 $4 \mathrm{~h}$ 样品 的 XRD 图谱

Figure $2 \mathrm{XRD}$ patterns of products obtained in $0.01 \mathrm{~mol} \cdot \mathrm{L}^{-1} \mathrm{HCl}$ (a) without calcination and calcined at (b) $600{ }^{\circ} \mathrm{C}$ or (c) $800{ }^{\circ} \mathrm{C}$ for $4 \mathrm{~h}$; XRD patterns of products obtained in $0.1 \mathrm{~mol} \cdot \mathrm{L}^{-1} \mathrm{NaOH}$ solution (d) without calcination and calcined at (e) $600{ }^{\circ} \mathrm{C}$, (f) $800{ }^{\circ} \mathrm{C}$ or (g) 1000 ${ }^{\circ} \mathrm{C}$ for $4 \mathrm{~h}$

板钛矿样品在不同的焙烧温度下晶型的转变情况 见图 $2 \mathrm{~d} \sim 2 \mathrm{~g}$. 图 $2 \mathrm{~d}$ 是在 $0.1 \mathrm{~mol} \cdot \mathrm{L}^{-1} \mathrm{NaOH}$ 中水热所得 锐钛矿样品的 XRD 图谱, 与粉末衍射标准卡片 JCPDS 29-1360(图 S4)相对应，说明产物为板钛矿晶型. 在 600 ${ }^{\circ} \mathrm{C}$ 下焙烧 $4 \mathrm{~h}$ 后(图 2e), 样品的 XRD 图谱与图 $2 \mathrm{~d}$ 相比, 衍射峰出峰位置无任何变化. 产物仍然为板钛矿晶型, 并且, 衍射峰峰强几乎保持不变, 说明板钛矿的热稳定 性较强. 在 $800{ }^{\circ} \mathrm{C}$ 下焙烧 $4 \mathrm{~h}$ 后(图 $2 \mathrm{f}$ ), 在 $2 \theta \approx 30.8^{\circ}$ 处 与板钛矿的(121) 晶面相对应的衍射峰大幅度下降, 仅 残留一个很微弱的衍射峰. 本来在 $2 \theta \approx 25.5^{\circ}$ 附近归属 于板钛矿(121)和(111)的两个衍射峰, 在图 $2 \mathrm{f}$ 中只剩下 
了一个, 并且峰强明显增加. 结合图 $2 \mathrm{f}$ 中在 $2 \theta \approx 37.8^{\circ}$ 、 $48.0^{\circ}$ 处归属于锐钛矿 $(004),(200)$ 晶面的典型特征峰, 我 们认为在 $2 \theta \approx 25.5^{\circ}$ 附近的主衍射峰应归属于锐钛矿的 (101)面. 此外, 在 $2 \theta \approx 27.5^{\circ}, 36.1^{\circ}$ 和 $41.2^{\circ}$ 的衍射峰与 金红石晶型的(110), (101), (111)相对应. 由上说明, 在 $800{ }^{\circ} \mathrm{C}$ 下板钛矿晶型几乎全部发生了晶型转变, 转变为 了锐钛矿和金红石晶相. 而在 $1000{ }^{\circ} \mathrm{C}$ 下焙烧 $4 \mathrm{~h}$ 的样 品(图 2g), 样品的 XRD 图谱显示样品已完全转变为纯 的金红石晶型. 从图 $2 \mathrm{e} \sim 2 \mathrm{~g}$ 可以发现, 板钛矿样品并没 有直接转变为金红石晶型, 很可能是先转变为锐钛矿晶 型, 然后又在高温下转变为了金红石晶型. 因此出现了 图 $2 \mathrm{f}$ 中的板钛矿、锐钛矿和金红石多种晶型共存现象.

二钛钛酸钠经过酸洗之后, 在不同的焙烧温度下晶 型的转变情况见图 3. 与图 $3 \mathrm{a}$ 相比, 图 $3 \mathrm{~b}$ 中 $2 \theta \approx 10^{\circ}$ 附 近表示层状结构层间信号的衍射峰明显降低, 变为一个 较宽的弱峰. 表明酸洗处理中, 二钛钛酸钠(图 3a)层间 的钠离子被氢离子所取代, 虽然转变为了对应的二钛钛 酸, 但是层状结构变形和剥离较为严重 ${ }^{[28]}$. 二钛钛酸在 $400{ }^{\circ} \mathrm{C}$ 下焙烧 $4 \mathrm{~h}$ 后(图 $3 \mathrm{c}$ ), $2 \theta \approx 10^{\circ}$ 附近表示层状结构 层间信号的衍射峰消失, $2 \theta \approx 25.3^{\circ}, 37.8^{\circ}, 48.0^{\circ}$ 的衍射 峰对应于锐钛矿(JCPDS 21-1272, 图 S4)的(101), (004), (200)晶面. 说明样品经 $400{ }^{\circ} \mathrm{C}$ 的焙烧处理后由层状钛 酸转变为锐钛矿晶型. 图 $3 \mathrm{~d}$ 显示样品在 $600{ }^{\circ} \mathrm{C}$ 下焙烧 4 $\mathrm{h}$ 的晶型信息, 与图 $3 \mathrm{c}$ 对比可知样品在 $600{ }^{\circ} \mathrm{C}$ 下焙烧处 理后依然保持锐钛矿晶型, 但是主峰 $2 \theta \approx 25.3^{\circ}$ 的峰强 明显增加, 半峰宽减小. 说明在 $600{ }^{\circ} \mathrm{C}$ 的焙烧温度下, 锐钛矿的晶粒尺寸有所增长, 结晶性明显提高. 而在

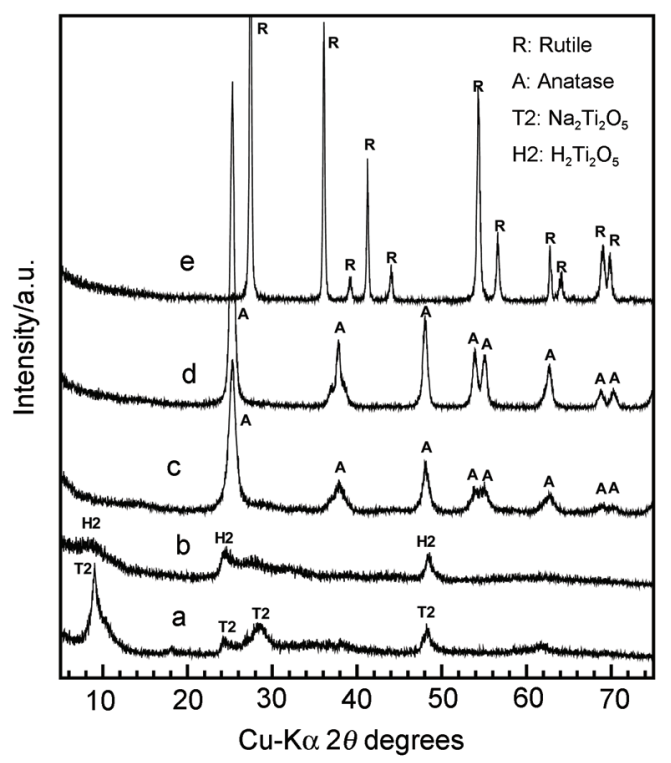

图 3 在 $1.0 \mathrm{~mol} \cdot \mathrm{L}^{-1} \mathrm{NaOH}$ 溶液中 (a)水热产物和(b)经酸洗处理后产 物的 XRD 图谱; 酸洗产物在(c) $400{ }^{\circ} \mathrm{C} 、$ (d) $600{ }^{\circ} \mathrm{C}$ 以及(e) $800{ }^{\circ} \mathrm{C}$ 焙 烧 $4 \mathrm{~h}$ 样品的 XRD 图谱

Figure 3 XRD patterns of products obtained in $1.0 \mathrm{~mol} \cdot \mathrm{L}^{-1} \mathrm{NaOH}$ solution (a) before acid-washing, (b) after acid-washing and then calcined at (c) $400{ }^{\circ} \mathrm{C}$, (d) $600{ }^{\circ} \mathrm{C}$ or (c) $800{ }^{\circ} \mathrm{C}$ for $4 \mathrm{~h}$
$800{ }^{\circ} \mathrm{C}$ 下焙烧处理 $4 \mathrm{~h}$ 后, 锐钛矿的衍射峰信号完全消 失，产物 XRD 图谱与金红石的粉末衍射标准卡片 JCPDS 21-1276(图 S4)相吻合. 说明产物在 $800{ }^{\circ} \mathrm{C}$ 的焙 烧条件下转变为纯的金红石晶型. 支持信息中的图 $\mathrm{S} 1$ 是样品在 $800{ }^{\circ} \mathrm{C}$ 下焙烧 $1 \mathrm{~h}$ 的 XRD 图谱, 与图 $3 \mathrm{e}$ 相对 比, 可以发现除了金红石的衍射峰之外, 在 $2 \theta \approx 25.3^{\circ}$ 处 仍保留一个较弱的衍射峰, 该衍射峰对应于锐钛矿的 (101)面. 由上说明在焙烧处理过程中, 钛酸并不能直接 转变为金红石晶型. 而是由钛酸先转变为锐钛矿, 之后 再次发生晶型转变, 转变为金红石晶型.

三钛钛酸钠经过酸洗之后，在不同的焙烧温度下晶 型的转变情况见图 4. 由图 $4 \mathrm{~b}$ 发现, 经过酸洗处理后, $2 \theta \approx 10^{\circ}$ 附近表示层状信号的衍射峰向高角度方向移动. 说明酸洗过程中, 层间的钠离子被库伦引力更强的氢离 子所取代, 致使层间距变窄 ${ }^{[29]}$. 图 $4 \mathrm{c}$ 是样品在 $400{ }^{\circ} \mathrm{C}$ 下焙烧 $4 \mathrm{~h}$ 的 XRD 图谱. 在 $2 \theta \approx 14.2^{\circ}, 28.6^{\circ}, 33.2^{\circ}$ 的衍 射峰对应于 $\mathrm{TiO}_{2}-\mathrm{B}$ 晶型(JCPDS 46-1238, 图 S4)的(001), (002)，(310) 晶面. 继续与粉末衍射标准卡片 JCPDS 46-1238(图 S4)进行对照, 发现其他衍射峰也都吻合良 好. 说明样品经 $400{ }^{\circ} \mathrm{C}$ 的焙烧处理后由层状钛酸转变 为 $\mathrm{TiO}_{2}-\mathrm{B}$ 晶型. 根据之前对锐钛矿和金红石的 XRD 图 谱分析, 我们可以得知, 在 $600{ }^{\circ} \mathrm{C}$ 下的焙烧样品为 $\mathrm{TiO}_{2}-\mathrm{B}$ 和锐钛矿的共存物; 在 $800{ }^{\circ} \mathrm{C}$ 和 $1000{ }^{\circ} \mathrm{C}$ 下的焙 烧样品分别是纯锐钛矿和纯金红石晶型. 说明三钛钛酸 钠经过酸洗之后，随着焙烧温度的逐渐升高，按照三钛 钛酸 $\mathrm{H}_{2} \mathrm{Ti}_{3} \mathrm{O}_{7} 、 \mathrm{TiO}_{2}-\mathrm{B}$ 、锐钛矿、金红石的顺序发生晶 型转变.

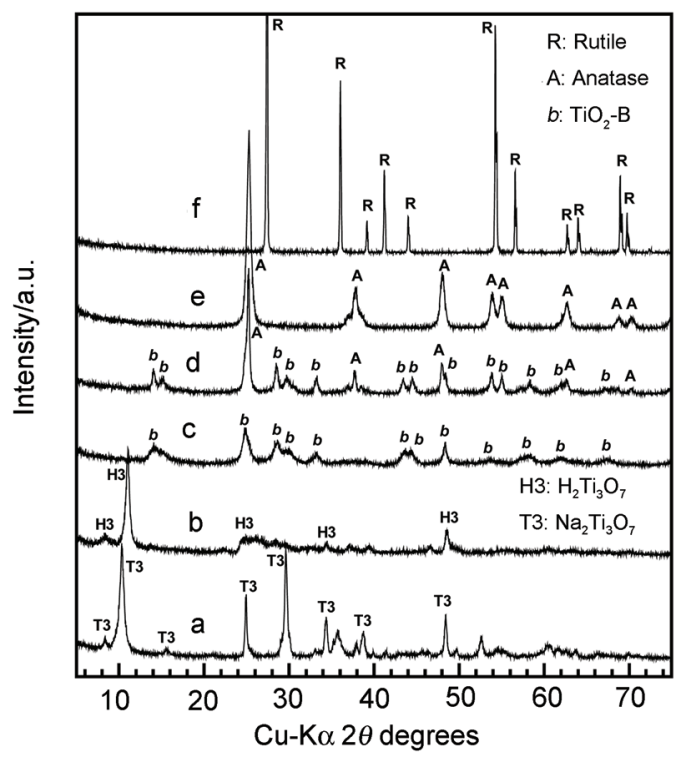

图 4 在 $8.0 \mathrm{~mol} \cdot \mathrm{L}^{-1} \mathrm{NaOH}$ 溶液中(a)水热产物和(b)经酸洗处理后产 物的 XRD 图谱; 酸洗产物在(c) $400{ }^{\circ} \mathrm{C}$, (d) $600{ }^{\circ} \mathrm{C}$, (e) $800{ }^{\circ} \mathrm{C}$ 以及(f) $1000{ }^{\circ} \mathrm{C}$ 焙烧 $4 \mathrm{~h}$ 样品的 XRD 图谱

Figure 4 XRD patterns of products obtained in $8.0 \mathrm{~mol} \cdot \mathrm{L}^{-1} \mathrm{NaOH}$ solution (a) before acid-washing, (b) after acid-washing and then calcined at (c) $400{ }^{\circ} \mathrm{C}$, (d) $600{ }^{\circ} \mathrm{C}$, (c) $800{ }^{\circ} \mathrm{C}$ or (c) $1000{ }^{\circ} \mathrm{C}$ for $4 \mathrm{~h}$ 


\section{2 形貌演化分析}

上述内容分析了金红石、锐钛矿、板钛矿、酸化的 钛酸盐等一系列不同晶型样品在不同焙烧温度下的晶 型转变行为. 随着晶型的转变, 这些样品在不同焙烧温 度下形貌演化行为见图 5 9.

图 5a 是 $3.00 \mathrm{~mol} \cdot \mathrm{L}^{-1} \mathrm{HCl}$ 中水热处理所得金红石样 品的 SEM 图像, 从图中可知金红石样品为纳米棒形貌. 纳米棒呈现单分散性, 其直径大约 $40 \mathrm{~nm}$, 大部分纳米 棒的长度在 $300 \sim 500 \mathrm{~nm}$ 之间, 少部分纳米棒长度较短. 虽然长度范围较宽, 但是直径较为均一. 从相对应的透 射电镜图像(图 5b)也证实了金红石样品为直径均一的纳 米棒形貌. 经过 $400{ }^{\circ} \mathrm{C}$ 焙烧后, 如图 5c 所示, 纳米棒的 直径增加到 $60 \mathrm{~nm}$ 左右, 长度有所变短, 长径比有所降 低, 并且纳米棒的端头处变得更为圆滑, 但是仍然呈现 良好的单分散性，仅有个别纳米棒相互烧结. 经过 600 ${ }^{\circ} \mathrm{C}$ 焙烧后, 如图 5d 所示, 金红石纳米棒端头或者侧边发 生了烧结, 与相邻的纳米棒熔融到一起. 但是, 纳米棒 的单根形貌依然可辨, 直径大约 $70 \sim 80 \mathrm{~nm}$, 长度大约 为 $300 \mathrm{~nm}$. 经过 $800{ }^{\circ} \mathrm{C}$ 焙烧处理后, 多数纳米棒已经发 生了较为严重的烧结现象, 如图 5e 所示, 在一大片烧结 到一起的纳米棒团簇中我们可以发现, 小片区域内数根 纳米棒完全熔融到一起，形成了更大尺寸的单晶区域， 纳米棒的形貌已经很难辨认. 但是, 根据团簇的表面形 貌, 依然可以指出烧结过程中纳米棒的排布与方向.

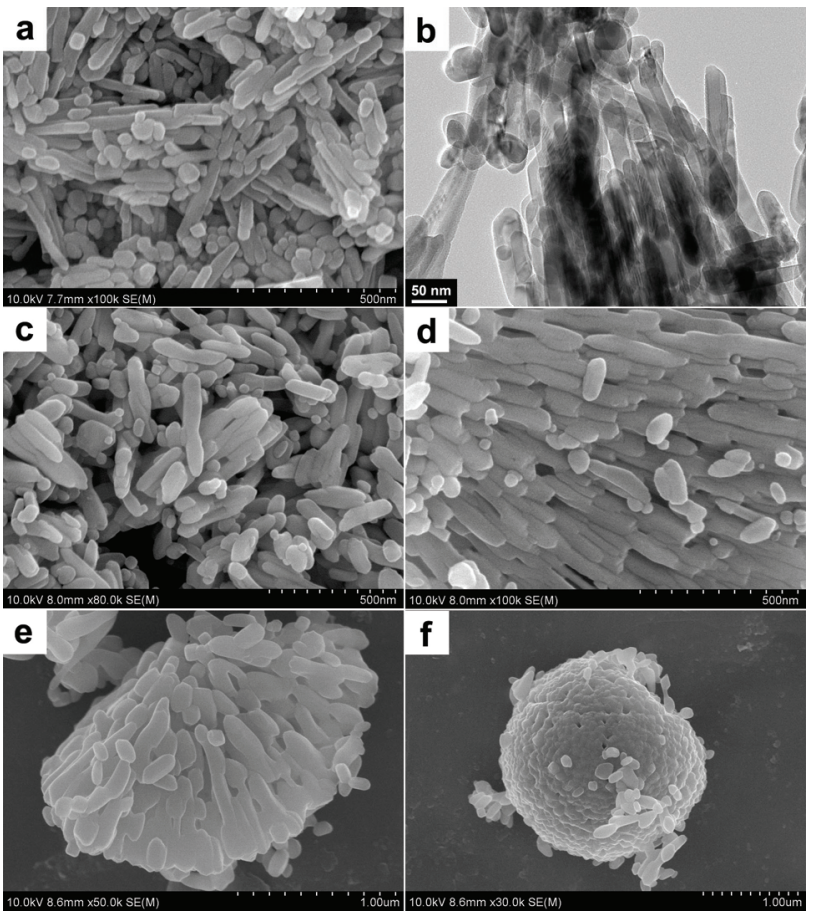

图 $53.00 \mathrm{~mol} \cdot \mathrm{L}^{-1} \mathrm{HCl}$ 中水热处理所得金红石样品的(a) SEM 和(b) TEM 图像, 以及该金红石样品在(c) $400{ }^{\circ} \mathrm{C} 、$ (d) $600{ }^{\circ} \mathrm{C} 、$ (e) $800{ }^{\circ} \mathrm{C}$ 和 (f) $1000{ }^{\circ} \mathrm{C}$ 下焙烧 $4 \mathrm{~h}$ 产物的 SEM 图像

Figure 5 (a) SEM and (b) TEM images of the rutile sample obtained in $3.00 \mathrm{~mol} \cdot \mathrm{L}^{-1} \mathrm{HCl}$ solution under hydrothermal treatment, and the SEM images of products calcined at (c) $400{ }^{\circ} \mathrm{C}$, (d) $600{ }^{\circ} \mathrm{C}$, (e) $800{ }^{\circ} \mathrm{C}$ and (f) $1000{ }^{\circ} \mathrm{C}$ for $4 \mathrm{~h}$
$1000{ }^{\circ} \mathrm{C}$ 焙烧处理后，纳米棒已经完全熔融成了一个表 面不平整的微球.

图 $6 \mathrm{a}$ 是 $0.01 \mathrm{~mol} \cdot \mathrm{L}^{-1} \mathrm{HCl}$ 中水热处理所得锐钛矿样 品的 SEM 图像, 从图中可知锐钛矿样品为单分散的纳 米颗粒. 颗粒尺寸大约为 $10 \mathrm{~nm}$ 左右, 粒径分布范围较 乍，并且分散性较好. 经过 $400{ }^{\circ} \mathrm{C}$ 焙烧后，如图 $6 \mathrm{~b}$ 所 示, 样品的粒径几乎保持不变, 仍为 $10 \mathrm{~nm}$ 左右. 但是, 纳米颗粒发生了轻微的团聚现象, 形成了直径在 60 $80 \mathrm{~nm}$ 的二次颗粒. 但是, 组成二次颗粒的锐钛矿一次 颗粒仍然是单独存在的，没有发生熔融现象。 $600{ }^{\circ} \mathrm{C}$ 下 焙烧后, 根据图 $2 \mathrm{~b}$ 的 XRD 图谱分析, 样品依然保持锐 钛矿晶型. 该样品的 SEM 图像见图 $6 \mathrm{c}$, 与图 $6 \mathrm{a}$ 和 $6 \mathrm{~b}$ 相比, $600{ }^{\circ} \mathrm{C}$ 焙烧样品的晶粒尺寸明显增大, 其粒径大 约为 $60 \mathrm{~nm}$ 左右. 说明在更高的焙烧温度下，小的锐钛 矿晶粒相互熔融, 晶间界面消失, 比表面积减小, 形成 了尺寸更大的二级单晶. 在晶体生长的过程中, 具有较 高表面能的晶面将优先被融合 ${ }^{[30]}$. 如果具有最高表面 能的晶间界面通过定向附着生长融合之后，原本具有次 高表面能晶间界面将成为新的最高表面能的晶间界面. 因此，随着焙烧温度的升高，次级晶间界面将继续相互 熔融 ${ }^{[30 ~ 32]}$. 因此，在 $800{ }^{\circ} \mathrm{C}$ 的焙烧温度下，见图 $6 \mathrm{~d}$, 大 尺寸的二级单晶颗粒也相互烧结在一起, 形成了微米级 的颗粒.

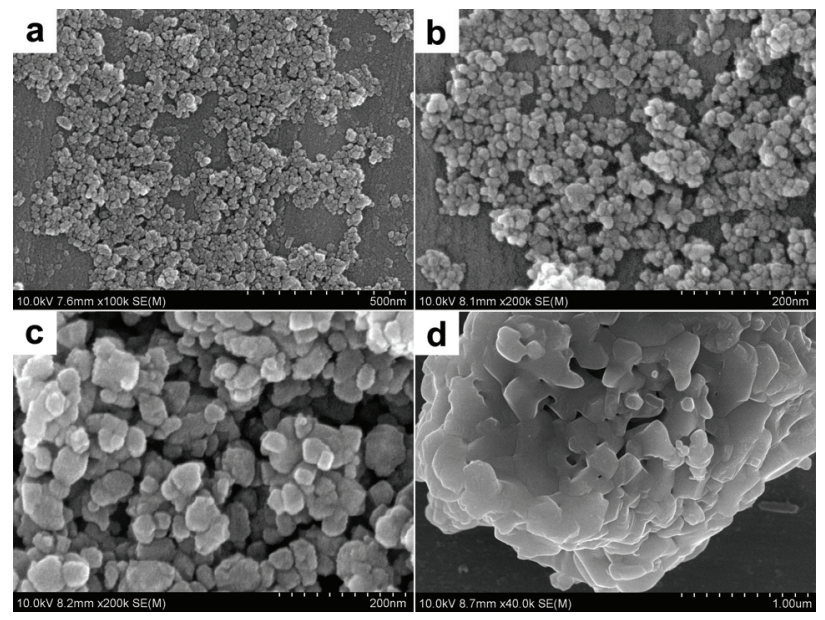

图 $60.01 \mathrm{~mol} \cdot \mathrm{L}^{-1} \mathrm{HCl}$ 中水热处理所得锐钛矿样品的(a) SEM 图像, 以及该锐钛矿样品在(b) $400{ }^{\circ} \mathrm{C} 、$ (c) $600{ }^{\circ} \mathrm{C}$ 和(d) $800{ }^{\circ} \mathrm{C}$ 下焙烧 $4 \mathrm{~h}$ 产 物的 SEM 图像

Figure 6 (a) SEM image of the anatase sample obtained in $0.01 \mathrm{~mol} \cdot$ $\mathrm{L}^{-1} \mathrm{HCl}$ solution under hydrothermal treatment, and the SEM images of products calcined at (b) $400{ }^{\circ} \mathrm{C}$, (c) $600{ }^{\circ} \mathrm{C}$ and (d) $800{ }^{\circ} \mathrm{C}$ for $4 \mathrm{~h}$

相对于金红石和锐针矿晶型而言，板铁矿晶型的晶 体结构更为复杂，也更难于合成 ${ }^{[33]}$. 在我们之前的工作 中, 我们也通过弱碱法合成了板钛矿 ${ }^{[19,20]}$. 之前也有一 些报道称合成了板钛矿 ${ }^{[34 ~ 37]}$. 但是，几乎无任何报道对 板钛矿在焙烧处理中的形貌演化行为进行研究. 本次工 作中, 我们对 $0.10 \mathrm{~mol} \cdot \mathrm{L}^{-1} \mathrm{NaOH}$ 下所得板钛矿样品在 焙烧处理中的形貌演化行为进行了研究. 图 $7 \mathrm{a}$ 和 $7 \mathrm{~b}$ 分 
别是未焙烧板钛矿样品的 SEM 和 TEM 图像. 与我们之 前的工作 ${ }^{[19]}$ 相类似地, 我们在合适浓度的 $\mathrm{NaOH}$ 中也得 到了纳米花形貌的板钠矿. 板钠矿纳米花由纳米棒所组 成, 每朵纳米花两端为放射状的多级结构花瓣, 纳米花 的中部为纳米棒团聚体. 每个纳米花簇的长度 (花瓣伸 展方向)尺寸大约在 $1 \mu \mathrm{m}$ 左右, 但纳米棒末梢的直径仅 有 $50 \mathrm{~nm}$ 左右. 经过 $400{ }^{\circ} \mathrm{C}$ 和 $600{ }^{\circ} \mathrm{C}$ 焙烧处理后, 如图 $7 \mathrm{c}$ 和 $7 \mathrm{~d}$ 所示, 纳米花的形貌几乎保持不变. 根据图 $2 \mathrm{e}$ 中的 XRD 图谱, 样品在 $600{ }^{\circ} \mathrm{C}$ 焙烧处理后其 XRD 的衍 射峰几乎保持不变. 说明板铁矿纳米花无论在晶型上还 是形貌上都具有较高的热稳定性. $800{ }^{\circ} \mathrm{C}$ 焙烧处理后 (图 7e), 纳米花的花瓣末梢变得较为圆滑, 并且长度缩 短, 尺寸变粗, 直径变为了 $150 \mathrm{~nm}$ 左右. 晶体之间并未 完全烧结, 仍留有较大的空隙, 变成了形貌特征不明显 的无序团聚体. $1000{ }^{\circ} \mathrm{C}$ 焙烧处理后, 如图 7f, 大多数纳 米花烧结在一起, 形成了尺寸达数微米的微球, 但是从 表面不平整的形貌依然可以观察到纳米花相互烧结的 痕迹.

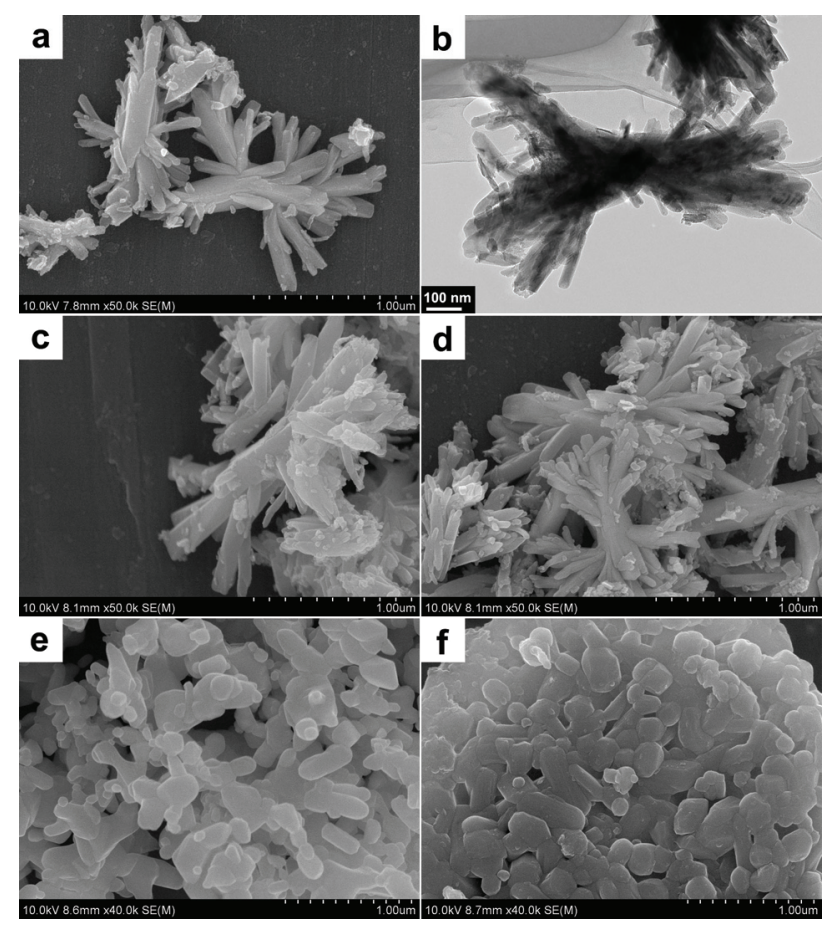

图 $70.10 \mathrm{~mol} \cdot \mathrm{L}^{-1} \mathrm{NaOH}$ 溶液中水热处理所得板钠矿样品的(a) SEM 和(b) TEM 图像, 以及该板钠矿样品在(c) $400{ }^{\circ} \mathrm{C}$, (d) $600{ }^{\circ} \mathrm{C}$, (e) 800 ${ }^{\circ} \mathrm{C}$ 和(f) $1000{ }^{\circ} \mathrm{C}$ 下焙烧 $4 \mathrm{~h}$ 产物的 SEM 图像

Figure 7 (a) SEM and (b) TEM images of the brookite sample obtained in $0.10 \mathrm{~mol} \cdot \mathrm{L}^{-1} \mathrm{NaOH}$ solution under hydrothermal treatment, and the SEM images of products calcined at (c) $400{ }^{\circ} \mathrm{C}$, (d) $600{ }^{\circ} \mathrm{C}$, (e) $800{ }^{\circ} \mathrm{C}$ and (f) $1000{ }^{\circ} \mathrm{C}$ for $4 \mathrm{~h}$

图 $8 \mathrm{a}$ 是 $1.00 \mathrm{~mol} \cdot \mathrm{L}^{-1} \mathrm{NaOH}$ 溶液中水热处理所得二 钛钛酸钠 $\mathrm{Na}_{2} \mathrm{Ti}_{2} \mathrm{O}_{5}$ 的 SEM 图像. 从图中可以观察到二 钛铁酸钠为薄片状, 大多数纳米片的长和宽大约为几百 纳米. 二钛钛酸钠经酸洗处理并在 $400{ }^{\circ} \mathrm{C}$ 下焙烧处理 后, 其 SEM 图像见图 8b. 与图 $8 \mathrm{a}$ 相比, 样品形貌的整
体趋势是长和宽变小，而厚度增加. 由二维的薄片形貌 向三维的颗粒形貌发展. 大多数颗粒的尺寸都减小到 $100 \mathrm{~nm}$ 以下. 说明在酸洗后 $400{ }^{\circ} \mathrm{C}$ 焙烧处理的条件下, 钛酸纳米片自发的朝向表面自由能降低的方向发生形 貌演化 ${ }^{[38,39]}$, 比表面积变小, 颗粒在三个维度上逐渐趋 于接近. $600{ }^{\circ} \mathrm{C}$ 焙烧处理后，见图 8c，尺寸在 $100 \sim 200$ $\mathrm{nm}$ 的颗粒相互团聚在一起, 形成了尺寸更大的团聚体. 部分颗粒已经相互烧结，形成了更大的单晶区域. 800 ${ }^{\circ} \mathrm{C}$ 焙烧处理后，见图 $8 \mathrm{~d}$ ，小尺寸的颗粒已经完全烧结在 一起, 形成了微米级的单晶颗粒.

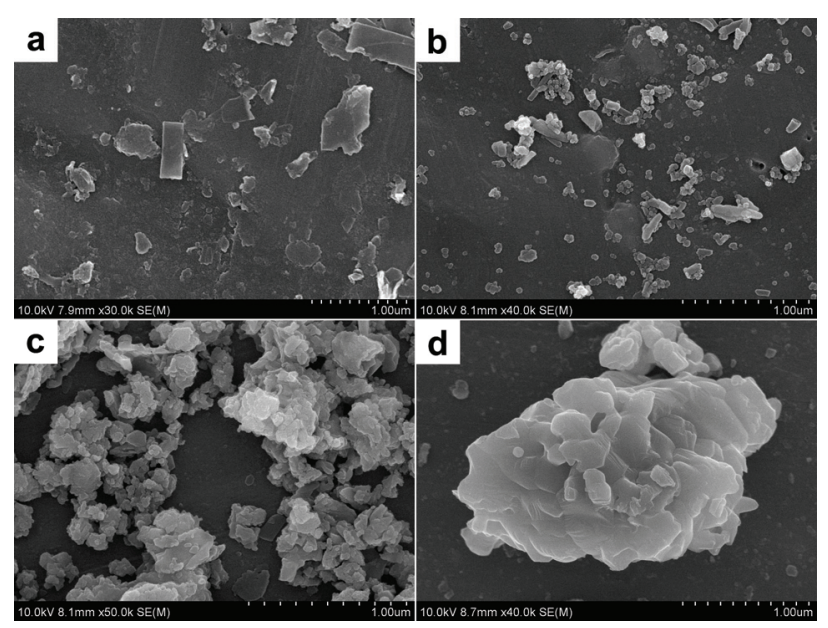

图 $81.00 \mathrm{~mol} \cdot \mathrm{L}^{-1} \mathrm{NaOH}$ 溶液中水热处理所得钛酸盐样品的(a) SEM 图像, 以及该钛酸盐样品酸洗处理后在(b) $400{ }^{\circ} \mathrm{C}$, (c) $600{ }^{\circ} \mathrm{C}$ 和(d) $800{ }^{\circ} \mathrm{C}$ 下焙烧 $4 \mathrm{~h}$ 产物的 SEM 图像

Figure 8 (a) SEM image of the titanate sample obtained in $1.00 \mathrm{~mol} \cdot$ $\mathrm{L}^{-1} \mathrm{NaOH}$ solution under hydrothermal treatment, and the SEM images of products after acid-washing and then calcined at (b) $400{ }^{\circ} \mathrm{C}$, (c) 600 ${ }^{\circ} \mathrm{C}$ and (d) $800{ }^{\circ} \mathrm{C}$ for $4 \mathrm{~h}$

图 $9 \mathrm{a}$ 是 $8.00 \mathrm{~mol} \cdot \mathrm{L}^{-1} \mathrm{NaOH}$ 溶液中水热处理所得三 钛铁酸钠 $\mathrm{Na}_{2} \mathrm{Ti}_{3} \mathrm{O}_{7}$ 的 SEM 图像, 可以观察到产物为纳 米线形貌, 纳米线的直径在 $70 \mathrm{~nm}$ 左右, 尺寸均一. 纳 米线长度有几百纳米, 甚至达到微米级. 经酸洗处理并 在 $400{ }^{\circ} \mathrm{C}$ 下焙烧处理后, 样品的 SEM 和 TEM 照片见图 $9 \mathrm{~b}$ 和 $9 \mathrm{c}$. 从图中可以观察到, 样品仍然为纳米线形貌, 但晶型已经转变为 $\mathrm{TiO}_{2}-\mathrm{B}$. 纳米线的直径仍然在 $70 \mathrm{~nm}$, 长度达到微米级, 但是有少量纳米线发生了轻微程度的 烧结. 从 9c 的 TEM 照片可以观察到纳米线的表面布满 了坑洼和空腔形貌. 这是因为三钛钛酸钠在经过酸洗之 后, 纳米线表面及内部形成了许多晶间裂缝 ${ }^{[29]}$. 在焙烧 过程中，通过晶体结构的重排与融合，小的晶间裂缝被 消除，大的晶间界空隙被进一步拉大，从而形成了特殊 的坑洼及空腔形貌 ${ }^{[29,40]}$. 经酸洗处理并在 $800{ }^{\circ} \mathrm{C}$ 下焙烧 处理后, 样品的 SEM 和 TEM 照片见图 9d 和 9e. 从图 中可以观察到, 多根纳米线沿同一方向排列, 并相互烧 结在一起, 形成了较粗的锐钛矿纳米棒, 每一束纳米棒 的直径大约在 $300 \sim 400 \mathrm{~nm}$ 之间, 由数十根纳米线所熔 融而成. 而经过 $1000{ }^{\circ} \mathrm{C}$ 焙烧的样品, 纳米线之间完全 
熔融, 并发生了不规则的剧烈形变, 形成了亚微米级的 金红石颗粒.

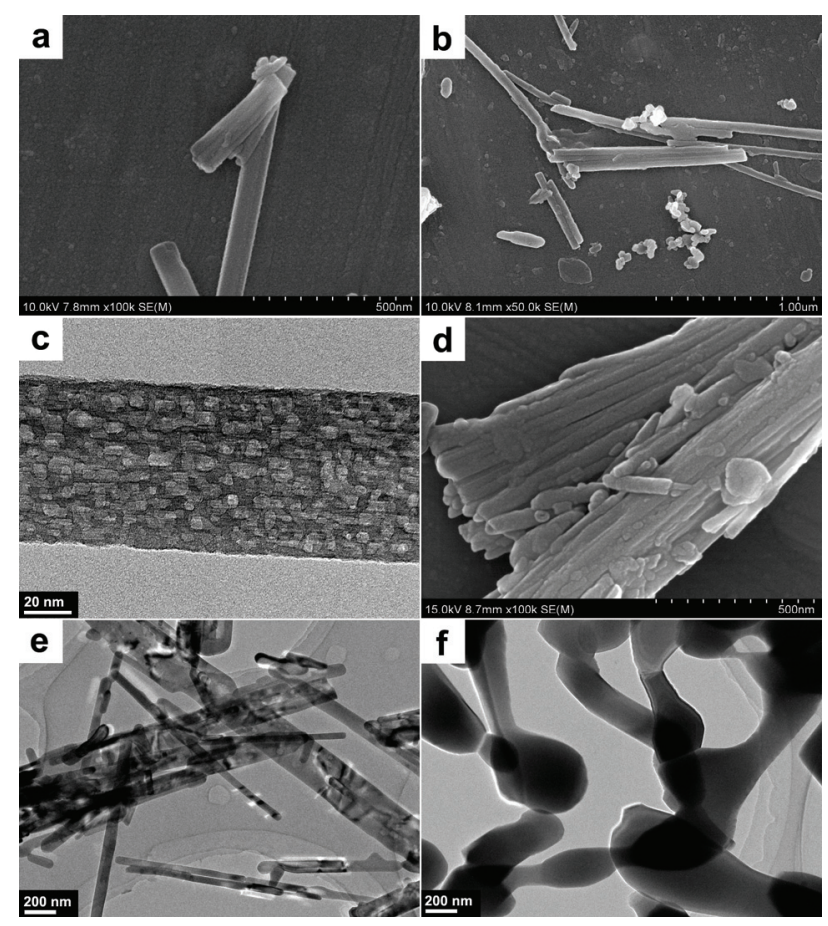

图 $98.00 \mathrm{~mol} \cdot \mathrm{L}^{-1} \mathrm{NaOH}$ 溶液中水热处理所得钛酸盐样品的(a) SEM 图像; 该钛酸盐样品酸洗处理后在 $400{ }^{\circ} \mathrm{C}$ 下焙烧 $4 \mathrm{~h}$ 产物的(b) SEM 和(c) TEM 图像, $800{ }^{\circ} \mathrm{C}$ 下焙烧 $4 \mathrm{~h}$ 产物的(d) SEM 和(e) TEM 图像以 及 $1000{ }^{\circ} \mathrm{C}$ 下焙烧 $4 \mathrm{~h}$ 产物的(f) TEM 图像

Figure 9 (a) SEM image of the titanate sample obtained in $8.00 \mathrm{~mol} \cdot$ $\mathrm{L}^{-1} \mathrm{NaOH}$ solution under hydrothermal treatment; (b) SEM and (c) TEM images of products after acid-washing and then calcined at $400{ }^{\circ} \mathrm{C}$; (d) SEM and (e) TEM images of products after acid-washing and then calcined at $800{ }^{\circ} \mathrm{C}$; (f) SEM image of product after acid-washing and then calcined at $1000{ }^{\circ} \mathrm{C}$

\section{3 形貌相图构建及晶体生长机理分析}

上述内容采用 XRD、SEM 和 TEM 等表征手段, 分 析了金红石、锐钛矿、板钛矿、酸化的钛酸盐等一系列 不同晶型样品在不同焙烧温度下的晶型转变和形貌演 化行为. 根据上述实验结果, 并考虑到水热酸碱浓度以 及焙烧温度两个因素对 $\mathrm{TiO}_{2}$ / 钛酸盐纳米材料晶型与形 貌变化影响, 我们绘制了如图 10 的晶相形貌演化示意 图.

根据图 10 分析可知, 较高的酸性条件对生成稳定 的金红石晶型有利. 在一个接近中性的酸碱浓度范围 内, 对生成锐钛矿晶型有利. 在一个较为合适的 $\mathrm{NaOH}$ 溶液浓度下, 即 $0.1 \mathrm{~mol} \cdot \mathrm{L}^{-1} \mathrm{NaOH}$ 溶液左右的一个较窄 的范围内, 水热处理产物为板钛矿晶型. 然后随着碱液 浓度的提高, 产物由板钛矿转变为二钛的钛酸钠, 进而 在更高的浓度下, 得到三钛的钛酸钠. 在不同的酸碱浓 度下, 得到的 $\mathrm{TiO}_{2} /$ 钛酸盐的晶型不同, 形貌也都不相 同，即: 金红石晶型的 $\mathrm{TiO}_{2}$ 纳米棒、锐钛矿晶型的 $\mathrm{TiO}_{2}$ 纳米晶粒、板钛矿晶型的 $\mathrm{TiO}_{2}$ 纳米花、二钛钛酸钠纳 米片和三钛钛酸钠纳米线. 二钛钛酸钠 $\mathrm{Na}_{2} \mathrm{Ti}_{2} \mathrm{O}_{5}$ 和三钛
钛酸钠 $\mathrm{Na}_{2} \mathrm{Ti}_{3} \mathrm{O}_{7}$ 经过酸洗处理之后, 分别转变为相对应 的二钛钛酸 $\mathrm{H}_{2} \mathrm{Ti}_{2} \mathrm{O}_{5}$ 和三钛钛酸 $\mathrm{H}_{2} \mathrm{Ti}_{3} \mathrm{O}_{7}$.

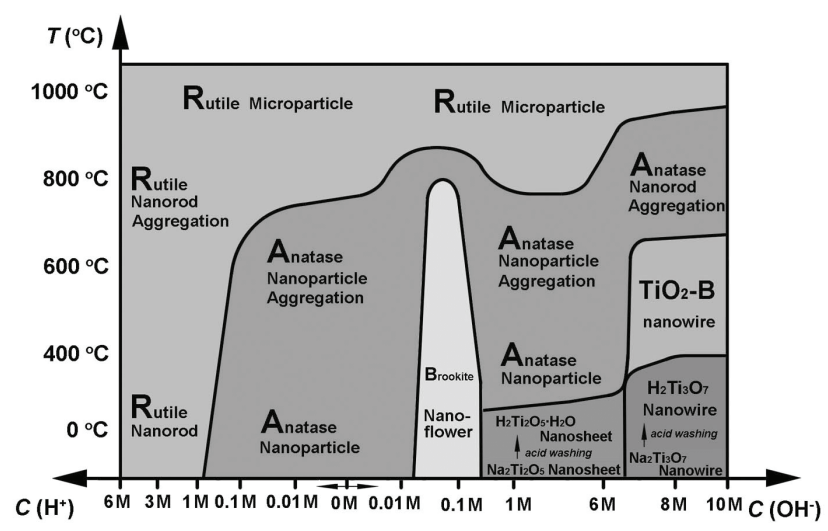

图 10 水热酸碱浓度以及焙烧温度两个因素对 $\mathrm{TiO}_{2} /$ 钛酸盐纳米材料 晶型转变及形貌演化的影响

Figure 10 Phase transition and morphological evolution behaviors of titania/titanate nanomaterials influenced by calcination temperature and hydrothermal acid and alkali concentration

上述 $\mathrm{TiO}_{2} /$ 钛酸盐随着焙烧温度的升高, 其晶型和 形貌都发生了不同程度和不同情况的转变. 其晶型转变 过程在很大程度上遵循奥斯特瓦尔德阶梯规 则 ${ }^{[41 \sim 43]}$ (Ostwald's step rule). 该规则认为, 晶体在生成 和生长过程中, 并不会直接生成最稳定的晶型; 而是在 外界能量的驱使下, 由亚稳态的晶型逐步向更加稳定的 晶型一步步转变, 所以在晶体中会存在多种晶型共存的 情况 ${ }^{[19,20,43 \sim 45]}$. 在二氧化钛的各种晶型中, 金红石是被 公认的最稳定的晶型, 其余晶型都是亚稳态的晶型. 据 文献报道，二氧化钛的晶型稳定性的排序为：金红石> 锐钛矿 $>$ 板钛矿 $>$ 其它晶型 ${ }^{[16,27,29]}$. 本次工作, 我们除 对上述 3 种晶型进行了热稳定性顺序考察之外, 还对 $\mathrm{TiO}_{2}-\mathrm{B}$ 晶型、二钛钛酸 $\mathrm{H}_{2} \mathrm{Ti}_{2} \mathrm{O}_{5}$ 和三铁铁酸 $\mathrm{H}_{2} \mathrm{Ti}_{3} \mathrm{O}_{7}$ 的 热稳定性进行了研究. 如图 10 所示, 根据我们的实验结 果, 我们发现在本次的实验条件下, 锐铁矿产物在超过 $600{ }^{\circ} \mathrm{C}$ 后开始发生晶型转变, 在 $800{ }^{\circ} \mathrm{C}$ 下已经完全转 变为金红石晶型. 板钛矿产物具有较好的热稳定性, 600 ${ }^{\circ} \mathrm{C}$ 下依然未发生晶型转变, 而在 $800{ }^{\circ} \mathrm{C}$ 的焙烧温度下, 板钛矿晶型转变为锐铁矿晶型, 并且由锐钛矿进而转变 为金红石晶型. 二钛钛酸 $\mathrm{H}_{2} \mathrm{Ti}_{2} \mathrm{O}_{5}$ 在 $400{ }^{\circ} \mathrm{C}$ 下已经完全 转变为锐钛矿晶型, 并且在 $800{ }^{\circ} \mathrm{C}$ 下, 由锐钛矿晶型进 而转变为金红石晶型. 三铁铁酸 $\mathrm{H}_{2} \mathrm{Ti}_{3} \mathrm{O}_{7}$ 的转晶行为最 为复杂, 在 $400{ }^{\circ} \mathrm{C}$ 下首先转变为 $\mathrm{TiO}_{2}$ - $\mathrm{B}$ 晶型, 进而在 更高的焙烧温度下转变为锐铁矿晶型, 最终在 $1000{ }^{\circ} \mathrm{C}$ 下转变为金红石晶型. 根据我们的实验结果，我们可以 同样从图 10 中获得了 $\mathrm{TiO}_{2}$ /钛酸(盐)的热稳定性强弱顺 序及相互之间的转晶行为, 该行为在很大程度上遵循奥 斯特瓦尔德阶梯规则.

$\mathrm{TiO}_{2} /$ 钛酸盐产物随着焙烧温度的升高, 其形貌也 发生了演化. 如图 10 所示, 随着焙烧温度的升高, 金红 石纳米棒的直径逐渐变粗, 纳米棒之间逐渐相互烧结, 
形成纳米棒团簇, 最终形成了微米级的颗粒; 锐钛矿纳 米晶粒粒径逐渐变大, 颗粒与颗粒之间相互团聚程度逐 渐加重, 形成锐钛矿纳米颗粒团聚体, 最终发生晶型转 变, 形成微米级的金红石颗粒. 板钛矿纳米花的热稳定 性较好, 到 $800{ }^{\circ} \mathrm{C}$ 时发生晶型转变时, 才随之发生了形 貌上的变化, 形成了由较粗的纳米棒构成的形貌特征不 明显的无序团聚体. 最终在 $1000{ }^{\circ} \mathrm{C}$ 的焙烧温度下也形 成了微米级的金红石颗粒. 二钛酸纳米片随着焙烧温度 的升高, 按照二钛酸纳米片、锐钛矿纳米颗粒、锐钛矿 纳米颗粒团聚体、金红石微米颗粒的顺序发生形貌变化. 无一例外的, 三钛酸纳米线随着焙烧温度的升高, 晶型 与形貌也发生了多级转变. 由三钛酸纳米线转变为 $\mathrm{TiO}_{2}-\mathrm{B}$ 纳米线、进而转变为锐钛矿纳米棒的团簇、最后 转变为金红石微米颗粒.

$\mathrm{TiO}_{2}$ / 钛酸盐产物随着焙烧温度的升高, 其形貌演 化过程符合经典热力学理论 ${ }^{[44]}$ 以及定向附着生长机 理 ${ }^{[31,45]}$. 在晶体生长及其形貌演化中最为基础的经典热 力学理论是 Gibbs-Curie-Wulff 晶体生长理论 ${ }^{[39,44]}$. 该理 论提出晶体在生长及演化过程中, 晶体的形貌由晶体晶 面的表面自由能决定, 晶体的最终形貌要保证体系总的 自由能最低. 在上述所有 $\mathrm{TiO}_{2}$ / 钛酸盐产物的形貌演化 过程中, 都是一个比表面积减小, 体系总表面自由能降 低的过程. 另外, 定向附着生长机理的特点是: 初级单 晶颗粒通过定向的晶面旋转、晶格重排、然后聚集并使 得晶粒取向一致化, 然后相同的晶面与晶面之间完全融 合, 在某个方向上定向附着从而形成一个较大的二级单 晶 ${ }^{[30]}$. 在本次工作中, 随着焙烧温度的升高, 金红石纳 米棒、锐钛矿纳米晶粒以及 $\mathrm{TiO}_{2}-\mathrm{B}$ 纳米线都是在某一 个特定晶面上首先发生融合，如金红石纳米棒和 $\mathrm{TiO}_{2}-\mathrm{B}$ 纳米线的融合面与纳米棒(线)的轴向方向平行, 形成纳米棒(线)束. 这些首先发生融合的晶面, 很大可 能性是所有晶面中表面能最高的. 这些晶面的优先融合 将导致总表面能的降低, 这样的一种方式驱动着定向附 着生长的进行. 如果具有最高表面能的晶间界面通过定 向附着生长融合之后，原本具有次高表面能晶间界面将 成为新的最高表面能的晶间界面. 因此, 随着焙烧温度 的升高, 次级晶间界面将继续进行定向附着生长 ${ }^{[30,31,45]}$. 最终, 得到了较大尺寸的单晶颗粒, 即金红石微米颗粒.

\section{3 结论}

本次工作对金红石、锐钛矿、板钛矿、酸洗处理后 的钛酸盐等一系列 $\mathrm{TiO}_{2}$ /钛酸产物进行焙烧处理, 系统 性地研究了焙烧温度的逐渐升高对产物晶型转变和形 貌演化的规律性影响. 并给出了水热酸碱浓度以及焙烧 温度两个因素对 $\mathrm{TiO}_{2}$ /钛酸盐纳米材料晶型与形貌影响 的二维形貌晶相示意图. 随着焙烧温度的升高, $\mathrm{TiO}_{2}$ /钛 酸纳米材料的晶型逐渐由亚稳态的晶型逐步向更加稳 定的晶型转变, 最终转变为最为稳定的金红石晶型, 这
种晶型转变行为在很大程度上遵循奥斯特瓦尔德阶梯 规则. 并且, 随着焙烧温度的升高, $\mathrm{TiO}_{2}$ /钛酸盐产物的 形貌演化过程中, 都是一个比表面积减小，体系总表面 自由能降低的过程, 其过程符合经典热力学理论以及定 向附着生长机理.

\section{4 实验部分}

\section{1 样品制备}

在快速搅拌下, 将 $8.5 \mathrm{~mL}$ 钛酸正四丁酯(TBOT)滴 加到 $75 \mathrm{~mL}$ 不同浓度的 $\mathrm{HCl}$ 或 $\mathrm{NaOH}$ 溶液中. 搅拌 15 $\min$ 后, 将混合均匀的悬浮液转入聚四氟乙烯内衬, 放 入反应釜中, 在 $180{ }^{\circ} \mathrm{C}$ 下水热 $24 \mathrm{~h}$. 水热结束后自然冷 却到室温, 之后倾去上层清液, 取出底部沉淀. 用去离 子水将沉淀物质洗涤数次, 直到 $\mathrm{pH} \approx 7$. 之后在红外线 干燥箱中在 $60{ }^{\circ} \mathrm{C}$ 左右干燥 $24 \mathrm{~h}$.

上述水热反应体系中，反应液的浓度为 $3.0 \mathrm{~mol} \cdot \mathrm{L}^{-1}$ $\mathrm{HCl} 、 0.01 \mathrm{~mol} \cdot \mathrm{L}^{-1} \mathrm{HCl} 、 0.1 \mathrm{~mol} \cdot \mathrm{L}^{-1} \mathrm{NaOH} 、 1.0 \mathrm{~mol} \bullet$ $\mathrm{L}^{-1} \mathrm{NaOH}$ 或 $8.0 \mathrm{~mol} \cdot \mathrm{L}^{-1} \mathrm{NaOH}$ 溶液. 所获得的 5 组样 品, 前 3 组样品无需进行酸洗处理. 后两组样品进行酸 洗处理的实验步骤如下: 在 $\mathrm{pH} \approx 1$ 的 $\mathrm{HCl}$ 溶液中摚拌 $12 \mathrm{~h}$, 然后用去离子水将酸洗后的样品洗涤数次, 直到 $\mathrm{pH} \approx 7$. 然后在红外线干燥箱中在 $60{ }^{\circ} \mathrm{C}$ 左右干燥 $24 \mathrm{~h}$.

焙烧处理实验操作: $0.01 \mathrm{~mol} \cdot \mathrm{L}^{-1} \mathrm{HCl}$ 水热样品、 1.0 $\mathrm{mol} \cdot \mathrm{L}^{-1} \mathrm{NaOH}$ 下水热并酸洗处理后样品在马弗炉中, 在 $400{ }^{\circ} \mathrm{C} 、 600{ }^{\circ} \mathrm{C}$ 和 $800{ }^{\circ} \mathrm{C}$ 的温度下焙烧 $4 \mathrm{~h}$, 自然冷 却到室温后得到焙烧产物. $3.0 \mathrm{~mol} \cdot \mathrm{L}^{-1} \mathrm{HCl}$ 水热样品、 $0.1 \mathrm{~mol} \cdot \mathrm{L}^{-1} \mathrm{NaOH}$ 水热样品、 $8.0 \mathrm{~mol} \cdot \mathrm{L}^{-1} \mathrm{NaOH}$ 下水热 并酸洗处理后样品在马弗炉中 $400{ }^{\circ} \mathrm{C} 、 600{ }^{\circ} \mathrm{C} 、 800{ }^{\circ} \mathrm{C}$ 和 $1000{ }^{\circ} \mathrm{C}$ 下焙烧 $4 \mathrm{~h}$, 自然冷却到室温后得到焙烧产 物. 焙烧处理是在空气中进行的, 升温过程从室温开始, 升温速率为 $2{ }^{\circ} \mathrm{C} / \mathrm{min}$. 每个样品每次焙烧处理的实验条 件严格保持一致.

\section{2 样品表征}

$\mathrm{X}$ 射线粉末衍射(XRD)分析: 将样品粉末分散在载 玻片上压实后置于日本 RIGAKU D/max-2600/PC 型 X 射线衍射仪上进行测试. 测试条件: 靶型 $\mathrm{Cu} \mathrm{K \alpha}(\lambda=$ $1.5406 \AA$ ), 工作电压 $40 \mathrm{kV}$, 电流 $100 \mathrm{~mA}$, 步长 $0.02^{\circ}$, 扫描速率 $4\left(^{\circ}\right) \cdot \mathrm{s}^{-1}$ 。

透射电镜(TEM)表征: 在 JEOL JEM 2100F 型场发 射透射电子显微镜上进行. 主要参数：加速电压 200 $\mathrm{kV}$, 点分辨率: $0.19 \mathrm{~nm}$, 线分辨率: $0.14 \mathrm{~nm}$. 制备样品 时, 取极少量的样品加入到离心管中, 然后加入无水乙 醇, 然后用超声波超声分散数分钟之后将离心管静置, 然后用一次性滴管取上层清液，垂直滴在具有碳支持膜 的铜网上, 以 $1 \sim 3$ 滴为宜. 将滴好样品的铜网低温干燥 后即可导入显微镜下观察.

场发射扫描电镜(FESEM)分析：FESEM 分析在 
HITACHI S-4800 型场发射扫描电子显微镜上进行, 加 速电压为 $10 \sim 15 \mathrm{kV}$. 测试前, 先将纳米级的金颗粒喷 酒在制备好的样品上，以增强样品的导电性.

\section{References}

[1] Guo, W. X.; Xu, C; Wang, X.; Wang, S. H.; Pan, C. F.; Lin, C. J.; Wang, Z. L. J. Am. Chem. Soc. 2012, 134, 4437.

[2] Jiang, D. W.; Zhou, T. S.; Sun, Q.; Yu, Y. Y.; Shi, G. Y.; Jin, L. T. Chin. J. Chem. 2011, 29, 2505.

[3] Zheng, Q.; Li, J. H.; Chen, Q. P.; Bai, J.; Zhou, B. X.; Cai, W. M. Chin. J. Chem. 2011, 29, 2236.

[4] Wan, Z. Q.; Zheng, S. N.; Jia, C. Y.; Yan, W. Acta Chim. Sinica 2009, 67, 403. (万中全，郑树楠，贾春阳，延卫，化学学报，2009, 67, 403.)

[5] Su, J. X.; Qu, W.; Ma, L. Y.; Yin, J.; Pan, Q. Acta Chim. Sinica 2008，66，2416. (苏继新，屈文，马丽媛，殷晶，潘齐，化学学报, 2008, 66, 2416.)

[6] Zhao, W. K.; Zhou, L.; Liu, C.; Hu, L.; Fang, Y. L.; Kiuchi, M. Acta Chim. Sinica 2003, 61, 699. (赵文宽, 周䂞, 刘昌, 胡领, 方佑龄, 木内正人, 化学学报, 2003, 61, 699.)

[7] Luan, X. N.; Guan, D. S.; Wang, Y. J. Phys. Chem. C 2012, 116, 14257.

[8] Zhao, B.; Chen, F.; Gu, X. N.; Zhang, J. L. Chem. Asian J. 2010, 5, 1546.

[9] Wu, H. B.; Lou, X. W.; Hng, H. H. Chem. Eur. J. 2012, 18, 2094.

[10] Yang, D. J.; Zheng, Z. F.; Zhu, H. Y.; Liu, H. W.; Gao, X. P. Adv. Mater. 2008, 20, 2777.

[11] Tsai, C. C.; Teng, H. Chem. Mater. 2006, 18, 367.

[12] Amano, F.; Yasumoto, T.; Shibayama, T.; Uchida, S.; Ohtani, B. Appl. Catal., B 2009, 89, 583.

[13] Shen, W. H.; Nitta, A.; Chen, Z.; Eda, T.; Yoshida, A.; Naito, S. J. Catal. 2011, 280, 161.

[14] Peng, Y. P.; Lo, S. L.; Ou, H. H.; Lai, S. W. J. Hazard. Mater. 2010, $183,754$.

[15] Scotti, R.; Bellobono, I. R.; Canevali, C.; Cannas, C.; Catti, M.; D’Arienzo, M.; Musinu, A.; Polizzi, S.; Sommariva, M.; Testino, A.; Morazzoni, F. Chem. Mater. 2008, 20, 4051.

[16] Li, J. G.; Ishigaki, T.; Sun, X. D. J. Phys. Chem. C 2007, 111, 4969.

[17] Morgan, D. L.; Zhu, H. Y.; Frost, R. L.; Waclawik, E. R. Chem. Mater. 2008, 20, 3800.

[18] Morgan, D. L.; Liu, H. W.; Frost, R. L.; Waclawik, E. R. J. Phys. Chem. C 2010, 114, 101.

[19] Zhao, B.; Chen, F.; Jiao, Y. C.; Zhang, J. L. J. Mater. Chem. 2010, 20,7990 .
[20] Zhao, B.; Chen, F.; Huang, Q. W.; Zhang, J. L. Chem. Commun. 2009, 34, 5115.

[21] Pavasupree, S.; Suzuki, Y.; Yoshikawa, S.; Kawahata, R. J. Solid State Chem. 2005, 178, 3110.

[22] Armstrong, A. R.; Armstrong, G.; Canales, J.; Bruce, P. G. Angew. Chem., Int. Ed. 2004, 43, 2286.

[23] Wu, Y. M.; Zhang, J. L.; Xiao, L.; Chen, F. Appl. Catal., B 2009, 88, 525.

[24] Kochkar, H.; Lakhdhar, N.; Berhault, G.; Bausach, M.; Ghorbel, A. J. Phys. Chem. C 2009, 113, 1672.

[25] Kuang, D. B.; Brillet, J.; Chen, P.; Takata, M.; Uchida, S.; Miura, H.; Sumioka, K.; Zakeeruddin, S. M.; Gräzel, M. ACS Nano 2008, 2, 1113.

[26] Wu, Y. M.; Liu, H. B.; Zhang, J. L.; Chen, F. J. Phys. Chem. C 2009, 113, 14689

[27] Hummer, D. R.; Kubicki, J. D.; Kent, P. R. C.; Post, J. E.; Heaney, P. J. J. Phys. Chem. C 2009, 113, 4240.

[28] Tsai, C. C.; Teng, H. Langmuir 2008, 24, 3434.

[29] Zhao, B.; Chen, F.; Qu, W. W.; Zhang, J. L. J. Solid State Chem 2009, 182, 2225

[30] Xu, H. L.; Wang, W. Z.; Zhu, W.; Zhou, L.; Ruan, M. L. Cryst. Growth Des. 2007, 7, 2720.

[31] Banfield, J. F.; Welch, S. A.; Zhang, H. Z.; Ebert, T. T.; Penn, R. L. Science 2000, 289, 751.

[32] Cho, K. S.; Talapin, D. V.; Gaschler, W.; Murray, C. B. J. Am Chem. Soc. 2005, 127, 7140.

[33] Jiao, Y. C.; Zhao, B.; Chen, F.; Zhang, J. L. CrystEngComm 2011 , 13, 4167.

[34] Yan, W. F.; Chen, B.; Mahurin, S. M.; Dai, S.; Overbury, S. H. Chem. Commun. 2004, 17, 1918.

[35] Buonsanti, R.; Grillo, V.; Carlino, E.; Giannini, C.; Kipp, T.; Cingolani, R.; Cozzoli, P. D. J. Am. Chem. Soc. 2008, 130, 11223.

[36] Tomita, K.; Petrykin, V.; Kobayashi, M.; Shiro, M.; Yoshimura, M.; Kakihana, M. Angew. Chem., Int. Ed. 2006, 45, 2378.

[37] Iskandar, F.; Nandiyanto, A. B. D.; Yun, K. M.; Hogan, Jr., C. J.; Okuyama, K.; Biswas, P. Adv. Mater. 2007, 19, 1408.

[38] Finnegan, M. P.; Zhang, H. Z.; Banfield, J. F. J. Phys. Chem. C 2007, 111, 1962.

[39] Ge, X.; Song, S. Y.; Zhang, H. J. CrystEngComm 2012, 14, 7306.

[40] Zhao, B.; Chen, F.; Liu, H. Q.; Zhang, J. L. J. Phys. Chem. Solids 2011, 72, 201.

[41] Santen, R. A. V. J. Phys. Chem. 1984, 88, 5768.

[42] Ostwald, W. Z Phys. Chem. 1897, 22, 289.

[43] Threlfall, T. Org. Process Res. Dev. 2003, 7, 1017

[44] Mullin, J. W. Crystallization, 4th ed., Butterworth Heinemann, Boston, 2001

[45] Penn, R. L.; Banfield, J. F. Science 1998, 281, 969. 